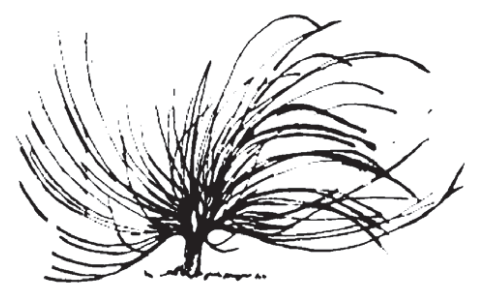

\title{
Transformación de las Bibliotecas Escolares a Centros de Recursos para el Aprendizaje: Retos y Obstáculos para el Profesional de la Información
}

\author{
Rosalyn Castrillo Sequeira ${ }^{1}$ \\ Universidad Nacional \\ Heredia, Costa Rica \\ rcastrillos@gmail.com
}

\begin{abstract}
Resumen
En este ensayo, se aborda la necesidad de convertir las bibliotecas escolares en centros de recursos para el aprendizaje que dinamicen el currículum de los centros educativos. Se mencionan políticas de gobierno donde se proyecta el fortalecimiento de las bibliotecas escolares costarricense y se destaca la experiencia chilena como modelo para esta transformación. Finalmente, se exponen algunos retos y obstáculos del profesional de la información ante este proceso de renovación.
\end{abstract}

Palabras claves: bibliotecas escolares, centros de recursos para el aprendizaje, bibliotecólogo pedagógico.

\begin{abstract}
In this essay, the author addresses the need to transform school libraries into resource centers for learning that
\end{abstract}

Recibido: 10 de febrero de 2013 - Aprobado: 20 de noviembre de 2013

1 Bachiller en Bibliotecología Pedagógica de la Universidad Nacional, Bachiller en Bibliotecología, Documentación e Información con énfasis en Tecnologías de la Información de la Universidad Nacional, Máster en Ciencias de la Educación con énfasis en Docencia de la Universidad Americana, actualmente estudiante de la Licenciatura en Bibliotecología Pedagógica de la Universidad Nacional. 
invigorate the curriculum of schools. Government policies are mentioned to project the strengthening of school libraries in Costa Rica, and the Chilean experience is highlighted as a model for this transformation. Finally, some challenges and obstacles of the information professional against the renewal process are dealt with.

Keywords: school libraries, resource centers for learning, pedagogic librarian.

\section{Introducción}

$\mathrm{E}$ 1 acontecer de la era de la información y del conocimiento trae consigo la necesidad de replantear la función de las bibliotecas escolares, siendo preciso trascender de los servicios bibliotecarios tradicionales, a servicios innovadores que se ajusten a las nuevas tendencias de la sociedad y que dinamicen el currículum de los centros educativos.

Autoridades del gobierno costarricense han contemplado esta necesidad, por lo cual, han incluido dentro del Plan Nacional de Desarrollo (PND) 2011-2014 y en las Líneas Estratégicas del Ministerio de Educación Pública (MEP), un proyecto para la modernización de las Bibliotecas Escolares mediante la transformación de estas a Centros de Recursos para el Aprendizaje (CRA) con acceso a tecnologías de la información y comunicación (TIC's).

En el Informe anual del cumplimiento de metas PND 2011 presentado por el Ministerio de Planificación Nacional y Política Económica (MIDEPLAN), se hace mención al compromiso del gobierno con este proyecto y a la continuidad que hasta el momento se le ha otorgado:

El proyecto \#000099 de nombre "Centros de Recursos para el Aprendizaje" se encuentra en etapa de ejecución, para el periodo 2011 se presupuestó el monto de $₫ 114.000 .000,00$ de fondos provenientes de presupuesto nacional. Se espera que el proyecto concluya la etapa de ejecución en el 2014. (MIDEPLAN, 2012, p. 93).

El liderazgo del proyecto lo ha asumido el Departamento de Bibliotecas Escolares y Centros de Recursos para el Aprendizaje (BEYCRA) del MEP, quienes se encargan de definir los pasos a seguir para alcanzar con éxito esta transformación. 
Es oportuno mencionar la experiencia del Sistema Educativo Chileno, en donde desde el año 1994 inician con el propósito de redimensionar el rol de las bibliotecas escolares chilenas, por lo que actualmente, la población educativa beneficiada con CRA alcanza "[...] el $95 \%$ de los establecimientos municipales y particulares subvencionados del país, las que corresponden a 10.472 en todo Chile (8.268 en básica y 2.204 en media). En total son más de 2.800 .000 alumnos beneficiados". (Ministerio de Educación de Chile, 2013, párrafo 3).

Esta exitosa experiencia está siendo considerada como modelo por el BEYCRA, y desde luego también debe ser estudiada por cada profesional a cargo de una biblioteca escolar; es por ello que se intentará definir en qué consiste el modelo de renovación de las bibliotecas escolares, con base en esta amplia experiencia chilena.

Iniciando con la naturaleza de un CRA, se puede decir, que consiste en una transformación del servicio tradicional de la biblioteca escolar mediante la incorporación de nuevos componentes que le permitan dinamizar el proceso educativo, así se menciona en el Manual para el CRA escolar del Ministerio de Educación de Chile:

[...] el lugar de la escuela y el liceo donde se reúne, en un espacio dinámico y de encuentro, una diversidad de recursos para el aprendizaje, que contienen información actualizada y apoya, a través de múltiples servicios, el proceso de enseñanza-aprendizaje. (Manual para el CRA escolar, 2009, p. 9).

En esta modalidad de biblioteca escolar, se integran diversidad de recursos y servicios que permiten innovar en aspectos como: el fomento a la lectura; el acceso a información actualizada; el desarrollo de colecciones en diferentes formatos; la incorporación de las TIC's; la educación de usuarios; la promoción de la curiosidad intelectual y la investigación en los estudiantes; la alfabetización informacional, entre otros.

Bajo este enfoque, se fortalece la estructura poco flexible centrada en el préstamo y resguardo de materiales impresos, para dar origen a un servicio promotor de conocimientos, que apoye en las distintas áreas del currículum y contribuya al mejoramiento de la calidad de la educación; esto mediante la generación de instancias de aprendizaje que transformen a la biblioteca escolar en un componente pedagógico importante, en un sitio de participación y de encuentro, en el que accionan 
en conjunto bibliotecólogos, docentes, directores, estudiantes, padres de familia y miembros de la comunidad.

Para lograr esta conversión es necesario añadir nuevos componentes en las bibliotecas escolares, así se afirma en el Manual para el CRA escolar:

Para llegar a hacer de una biblioteca un Centro de Recursos para el Aprendizaje, es necesario revalorar y repensar cada uno de los elementos que la componen: el espacio, la colección, y el equipo de trabajo, los que en conjunto deben alcanzar un funcionamiento armónico y eficiente. (Manual para el CRA escolar, 2009, p. 11).

El profesional de la información debe asumir el desafío de integrar los componentes que más se ajusten a las necesidades de sus usuarios y a la realidad en que se encuentra inmersa su biblioteca. Algunos de los componentes sugeridos se detallan a continuación:

\section{Espacio}

Un lugar agradable que atraiga a los usuarios a frecuentar y hacer uso de la biblioteca, idealmente con colección abierta, para brindar un mayor acceso a los recursos.

Con áreas delimitadas para estudio individual, trabajos grupales, computadoras, audiovisuales, colección y circulación; y que estos espacios cuenten con óptima iluminación, ventilación y colores que favorezcan el aprendizaje.

La posición del CRA dentro de la institución debe ser estratégica, alejada de zonas ruidosas, estipulando el cumplimiento de la Ley 7600 para personas con discapacidad y considerando normas de seguridad para la protección de los usuarios, personal y bienes materiales.

\section{Colección Bibliográfica}

Corresponde al conjunto de recursos informativos seleccionados de acuerdo con los programas de estudio para apoyar el proceso de enseñanza-aprendizaje. Como parte de estos recursos se pueden mencionar: libros, materiales de referencia, publicaciones periódicas, mapas, audiovisuales, sitios web, juegos y cualquier otro material, ya sea en 
formato impreso, digital o electrónico, que contribuya en el proceso formativo y recreativo del estudiante.

\section{Tecnologías de la Información y la Comunicación}

En la actualidad se cuenta con diversidad de herramientas tecnológicas que se pueden incorporar en las escuelas y colegios para renovar las prácticas educativas. El CRA debe ser promotor en esta asociación, haciendo uso de aplicaciones colaborativas de la web 2.0, tales como blogs, redes sociales, wikis, foros, páginas web, chats, correo electrónico, portales educativos, entre otros.

La tendencia actual es contar con plataformas que permitan virtualizar los servicios bibliotecarios, por tanto estas herramientas se convierten en grandes aliados para la promoción de los servicios del CRA, para compartir y producir información de manera colaborativa y para llegar a la mayor cantidad de usuarios sin limitaciones por distancia o tiempo.

Las bibliotecas deben combatir la brecha digital, por ello necesitan contar con equipo tecnológico y conectividad, disponible tanto para la parte de gestión como para los usuarios. Asimismo, en el CRA se deben brindar programas de capacitación en cuanto al uso de estos recursos.

\section{Administrativo}

La labor esencial para la gestión de un CRA se centra en la creación de servicios y productos acordes con las necesidades educativas de los usuarios, por ello de acuerdo con el Manual para el CRA escolar (2009, p. 27-28), esta gestión debe dividirse en tres áreas fundamentales:

- Gestión administrativa: corresponde a todas las medidas para lograr una organización efectiva, tanto en lo que respecta a la parte de generación de recursos como al manejo que se haga de los materiales bibliográficos. Esta gestión incluye aspectos como: desarrollo de la colección, inscripción, sellado, etiquetado, procesos técnicos y mecanismos para la circulación de materiales.

- Gestión pedagógica: se refiere a la coordinación docente-biblioteca y a las acciones para integrar la biblioteca al currículo del centro educativo. Este componente incluye: formación de usuarios, difusión de la información (boletines, exposiciones, alertas), 
planes de fomento a la lectura, actividades culturales, alfabetización informacional, programas para el desarrollo de habilidades investigativas, creación de espacios recreativos, concursos, debates, charlas, talleres, promoción de la interdisciplinariedad, entre otros.

- Redes de trabajo: consiste en establecer lazos de cooperación para intercambiar recursos con los demás miembros de la comunidad educativa. Un CRA necesita de un verdadero compromiso del bibliotecólogo, además de un trabajo en equipo con docentes y director de la institución. El bibliotecólogo debe ser el líder de este equipo y convencer al cuerpo docente acerca de la importancia de la coordinación docente-biblioteca en pro de la mejora del proceso educativo. El apoyo de la parte administrativa es primordial para que desde su posición dicte políticas, haga gestiones y fiscalice tanto la labor del bibliotecólogo como la disposición que muestren los docentes.

\section{Recursos humanos}

El bibliotecólogo ya no debe ser visto como un simple administrador del local y de los recursos materiales que se encuentran dentro de la biblioteca, sino que pasa a ser parte activa del proceso formativo, mediante la realización de las distintas labores del componente administrativo antes mencionadas.

El bibliotecólogo no contribuye a mejorar el proceso de enseñanzaaprendizaje cuidando exámenes o asumiendo otras labores administrativas ajenas a la biblioteca; en este sentido, el profesional de la información debe hacer respetar su profesional, generando cambios que conviertan a la biblioteca en un lugar atractivo y necesario en el centro educativo.

\section{Algunos Retos y Obstáculos para el Profesional de la Información}

Una vez conocidos los componentes básicos de un CRA, es posible confrontar la realidad en la cual se encuentran inmersas muchas bibliotecas escolares del país y que se señalen algunos retos y obstáculos que puede encontrar el profesional de la información en su labor.

Se puede afirmar, de acuerdo con la óptica de la experiencia, que asumir la gestión de una biblioteca escolar en el sistema de educación 
pública costarricense, es un gran desafío, sobre todo si se considera todos los inconvenientes que por sí solo presenta el sistema.

La primera dificultad tiene que ver con el tema económico, ya que no en todos los centros educativos se cuenta con un monto designado dentro del presupuesto que sea de uso exclusivo para la biblioteca.

Por ello, es que las fuentes para el desarrollo de la colección se limitan a las donaciones de editoriales o que los mismos estudiantes y profesores realizan, además de los envíos que esporádicamente hace el BEYCRA; sin embargo, estas formas de adquisición son insuficientes, pues por lo general, se reciben materiales repetidos o que no resuelven las principales necesidades informativas de los usuarios.

Las compras de materiales se efectúan sólo en el momento que la dirección de la institución en conjunto con la Junta Administrativa deciden designar presupuesto, o cuando el bibliotecólogo con el comité de biblioteca realizan actividades para generar estos recursos. De ahí que una de las mayores debilidades de las bibliotecas escolares se relaciona con lo insuficientes y desactualizados materiales que poseen.

El espacio es otra polémica constante en las bibliotecas. Dado los problemas de infraestructura educativa que se viven en el país, es que en la mayoría de las instituciones se destinan espacios muy pequeños para ubicar la biblioteca, lo cual hace imposible la delimitación de áreas y la realización de algunas actividades. Además, ante la falta de aulas, en ocasiones las bibliotecas se convierten en lugares improvisados para impartir lecciones y formalizar reuniones.

En cuanto al acceso a las TIC's, nuevamente se pone en jaque el tema del presupuesto, sobre todo porque muchas bibliotecas no disponen ni siquiera de un computador con acceso a internet para el trabajo bibliotecario. Entonces, ¿Cómo hacer posible la virtualización de los servicios bibliotecarios y el desarrollo de colecciones hibridas?; ¿Cómo atraer a usuarios inmersos en el mundo de la tecnología?; y sobre todo, ¿Cómo convertir a la biblioteca escolar en un medio para disminuir la brecha digital, cuando muchas están formando parte de ella?

Aunque el proyecto de gobierno para la creación de los CRA contempla la dotación de equipo tecnológico, el problema es que lo hará únicamente con el 35\% de las bibliotecas escolares del país. Sin menospreciar este esfuerzo, es lamentable su carácter excluyente sobretodo porque se está ensanchando aun más la brecha que ya existe entre las mismas bibliotecas escolares; por esto, es importante la continuidad de políticas 
que apoyen el fortalecimiento de las bibliotecas escolares, la dotación de presupuestos para la adquisición de recursos, pero sobre todo la implantación de un cambio de mentalidad en el sistema educativo costarricense, para brindar a la biblioteca escolar o CRA, el reconocimiento que merece como agente de promotor de cultura y conocimiento.

Ante este panorama, queda en manos del profesional de la información asumir el gran reto de transformar su biblioteca, trabajando de manera eficiente y efectiva para sacar el máximo provecho de los pocos o muchos recursos que se tengan, sin duda la calidad y la excelencia siempre deben orientar su servicio.

\section{Conclusiones}

A lo largo de este ensayo, se han comentado aspectos referentes a la necesidad de renovar las bibliotecas escolares y la existencia de políticas gubernamentales que pretenden su modernización. Igualmente, se han señalado componentes del modelo chileno que deben estar presentes en esta conversión, a partir de los cuales, se han determinado algunos retos y obstáculos para la gestión de bibliotecas escolares costarricenses; por esto, a modo de desenlace se presentan algunos aspectos que se deben repasar como parte de la misión profesional de transformar las bibliotecas escolares a CRA:

- Las bibliotecas escolares enfrentan tiempos de cambio, el profesional de la información tiene el desafío de innovar los servicios de acuerdo con los nuevos requerimientos de usuarios inmersos en un mundo donde abunda la tecnología y la información, por ello las bibliotecas deben ofrecer una amplia oferta de servicios y productos informativos que promuevan el aprendizaje en todas las áreas formativas.

- La transformación de las bibliotecas escolares en CRA, deben posicionarlas como una atracción educativa para los estudiantes y un insumo necesario en el proceso de aprendizaje, para lo cual se requiere dejar atrás la tradicional concepción del servicio de biblioteca destinado solamente al repositorio y préstamo de libros. Un verdadero CRA, necesita la incorporación de componentes que le permitirán brindar un servicio más dinámico para el desarrollo del currículo. 
- $\quad$ El bibliotecólogo debe trabajar en un cambio de mentalidad profundo en cuanto a lo que se espera de la biblioteca en un centro educativo. Entre más promoción se brinde del potencial que representa contar con un CRA, mayores pueden ser las exigencias que se le hagan y también mayores las posibilidades de que el docente incluya dentro de sus planeamientos, los recursos y servicios de la biblioteca.

- Todos los componentes del CRA son indispensables para hacer de la biblioteca un sitio moderno y participativo, que apoye en contenidos pero también en el desarrollo de competencias y habilidades para la vida; así como en el aprendizaje continuo por medio de los programas de fomento a la lectura.

- $\quad$ Bajo el modelo de gestión CRA se debe brindar apoyo planificado al proceso educativo, mediante la programación de actividades que hagan de la biblioteca un pilar fundamental en el desarrollo del pensamiento crítico y en la generación de aprendizajes significativos.

- $\quad$ El proyecto de gobierno de creación de CRA tiene pretensiones muy buenas para el fortalecimiento de las bibliotecas escolares, sin embargo por su carácter excluyente soluciona solo a medias muchos de los problemas existentes en el sistema, es de esperar que futuros gobernantes crean en este proyecto y le brinden la continuidad que merece, principalmente por su gran aporte en el mejoramiento de la calidad de la educación costarricense. Se deben superar debilidades a nivel del sistema educativo, asumir obstáculos como desafíos profesionales y considerar experiencias exitosas como los modelos de CRA chilenos, fuente de inspiración para orientar la gestión de las bibliotecas escolares costarricenses. 


\section{Referencias bibliográficas}

García, M. (2005). La biblioteca escolar como centro de recursos para el aprendizaje (CRA). Recuperado de http://doteine.uc3m.es/docs/cuevascerverocive.pdf

Ministerio de Educación de Chile. (2013). Ministra de Educación lanza campaña para fomentar la lectura yel uso de las bibliotecas escolares. Recuperado de http://www. mineduc.cl/index2.php?id_portal=1\&id_seccion=10\&id_contenido $=25577$.

Ministerio de Educación de Chile. (2012). Bibliotecas escolares / CRA. Historia. Recuperado de http://www.bibliotecas-cra.cl/quienes/quie_hist.html

Ministerio de Educación de Chile. (2011). Bibliotecas escolares / CRA. Cobertura. Recuperado de http://www.bibliotecas-cra.cl/quienes/quie_cober.html

Ministerio de Educación de Chile. (2009). Manual para el CRA Escolar: por una biblioteca moderna y dinámica. Recuperado de http://www.bibliotecas-cra.cl/ uploadDocs/ManualCRAEscolar.pdf

Ministerio de Planificación Nacional y Política Económica de Costa Rica. (2012). Informe anual cumplimiento de metas PND 2011. Recuperado de

http://documentos.mideplan.go.cr/alfresco/d/d/workspace/SpacesStore/d37121113335-43d5-974b-2b9a861f1732/informe_anual_de_avance_de_las_metas.pdf 